\title{
Adaptive Testing of Ferromagnetic Materials Utilizing Barkhausen Noise
}

\author{
J. PAL'A AND J. BYDŽOVSKÝ \\ Slovak University of Technology in Bratislava, Institute of Electrical Engineering \\ Ilkovičova 3, 81219 Bratislava, Slovak Republic \\ (Received March 6, 2014; in final form April 16, 2014)
}

\begin{abstract}
A new method of the Barkhausen noise measurement and optimization of the measured data processing with respect to non-destructive evaluation of ferromagnetic materials is proposed. The measuring method is based on well-known method of magnetic adaptive testing where a complex set of minor hysteresis loops is measured. Collecting the sets of obtained Barkhausen noise envelopes into suitable designed matrices and optimizing the data processing with respect to the maximum sensitivity to the evaluated variable were developed. The method is illustrated on the evaluation of plastic deformation of a steel sample. It is shown that the proposed measuring method and measured data processing bring an improvement of the sensitivity to the evaluated variable when comparing with measuring the traditional Barkhausen noise parameter (root mean square).
\end{abstract}

DOI: 10.12693 /APhysPolA.126.855

PACS: 81.40.Rs, 75.60.Ej

\section{Introduction}

The Barkhausen noise (BN) yields statistical information about magnetization process in ferromagnetic materials at a slowly changing applied magnetic field. Because it originates from discontinuous movements of domain walls, it reflects the interaction of domain walls with structural defects, such as grain boundaries, dislocations, local mechanical stresses, inclusions, and precipitates. Magnetization processes are closely linked not only to these structural defects, but also to the other properties of the sample, such as the uniformity, texture, and the sample shape. The BN measurements are therefore widely used as nondestructive evaluation techniques for inspection of ferromagnetic materials, including the assessment of dislocation density [1], grain size [2], recovery and recrystallization [3], fatigue [4], and effects of applied stresses [2].

The BN is usually measured using a yoke exciting the sample to saturation. However, the influence of structural changes and material properties on $\mathrm{BN}$ in general varies throughout the magnetization process, thus the parameters of BN are, among others, dependent on the amplitude of the magnetic field [5]. To do the BN measurements in a comprehensive way, for example for later usage when the samples are not already accessible, there is the question of at what field amplitudes the $\mathrm{BN}$ should be measured. Moreover, application of measurements of the BN for in situ structural changes characterization of steels relies on optimization of sensitivity to structural changes, which includes selection of the optimum magnetic field amplitude.

In this paper we propose to collect the measurements of the BN with a stepwise increased amplitude of the magnetic field, employing similar approach as in the method of magnetic adaptive testing (MAT) [6]. This method is based on measurement of parameters of a set of minor hysteresis loops. It is similar to the method used to collect the magnetization data for identification of the Preisach distribution function (the Preisach model of hys- teresis). In contrary, the traditional magnetic hysteresis methods utilize measurement of a few major's loop parameters, such as coercive field, maximum permeability, and remanent magnetic induction [7]. These parameters are very well suited for characterization of magnetic properties of ferromagnetic materials, but they are usually not optimized for assessment of structural properties of materials. On the other hand, the magnetic adaptive method yields parameters (e.g. differential permeability) of any of the point on any of the minor loops, from which we choose the parameter that is most sensitive to the investigated property change and thus we can adapt the method to the investigated material and property [6].

\section{Description of the method}

The MAT method of testing ferromagnetic materials utilizes systematic measurement of families of minor hysteresis loops at the field amplitudes increasing stepwise from a minimum value up to the saturation, conducted on samples of the examined material subjected to the investigated structural change. For each structural stage of the sample, a matrix of the measured differential permeability or the other magnetic variable is arranged. Matrices of all structural stages have the same dimensions, with rows and columns representing the applied field and the field amplitude. The sensitivity of each matrix element to the investigated structural change is calculated and the most sensitive elements are used for evaluation of structural changes. The method was used with success, e.g. for the assessment of plastic deformation [8], embrittlement [9], decarburization of surface [10], and flaws [11].

Utilizing similar approach as in the MAT, the measurement of a set of BN signals obtained at stepwise increasing field amplitudes is proposed. The BN is measured in an applied field, $h_{\mathrm{A}}$, which is varied within amplitudes, $\pm h_{\mathrm{M} j}$ with the fixed field rate (see Fig. 1a). After demagnetizing the sample, the first period of the triangular field with an initial amplitude, $h_{\mathrm{M} 1}$, is generated. Then 
the next periods of the field are generated, the amplitude of each period being the amplitude of the previous period plus one experimentally predefined step of the amplitude, $h_{\mathrm{M} j+1}=h_{\mathrm{M} j}+\Delta h_{\mathrm{M}}$. When the field amplitude exceeds the predefined limit, the measurement is completed. In addition to this type of magnetizing known from the MAT, we tested also magnetizing with pauses between individual periods of the field (Fig. 1b). During these pauses, the measurement of the BN is stopped, the results are saved and the sample is demagnetized to reach the same initial magnetic state. So in this case, we need the memory of data acquisition system for storing the BN only during one period of the field. This has advantage of lower requirements on the memory of data acquisition system, which needs to store much more data in comparison to the hysteresis loops measurements.

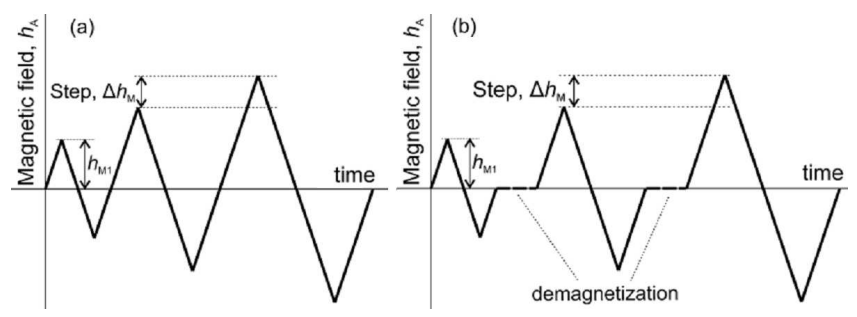

Fig. 1. The triangular wave forms of the applied field without (a) and with (b) pauses between individual periods.

The recorded quantities are arranged in data files with four columns for each field amplitude $h_{\mathrm{M} j}$. The first column is the measured time, the second is the field $h_{\mathrm{A}}$, the third is the $\mathrm{BN} v_{\mathrm{B}}$, and the fourth one is the envelope of the $\mathrm{BN} v$ calculated from the third column signal. The envelope of the BN voltage is obtained by calculating the root mean square (RMS) value within a moving window

$$
v_{k}=\sqrt{\frac{1}{N} \sum_{j=k}^{k+N-1} v_{\mathrm{B} j}^{2}},
$$

where $N$ is the number of adjacent samples of the BN voltage used for calculating the $k$-th point of the envelope and $v_{\mathrm{B} j}$ is the $j$-th sample of the $\mathrm{BN}$ voltage.

The data files contain comprehensive information about magnetization process in the sample and can be used e.g. for assessing the most sensitive region of the magnetization process in $\left(h_{\mathrm{A}}, h_{\mathrm{M}}\right)$ plane, for theoretical analyses of the magnetization process, etc. In order to evaluate the sensitivity to the investigated structural change, it was proposed to assembly the $V$-matrices from the recorded envelopes of the $\mathrm{BN}$ only for half of each period of the field ranging from the maximum positive value to the negative maximum, with elements $v_{i j}$ corresponding to the $\left(h_{\mathrm{A} i}, h_{\mathrm{M} j}\right)$-field pairs

$$
V=\left[\begin{array}{ccccc} 
& & & v_{1, m} \\
& & & \bullet & v_{2, m} \\
& & \bullet & & \bullet \\
& v_{c-r_{2}, 2} & & \bullet \\
v_{c-r_{1}, 1} & \bullet & & \bullet \\
\bullet & v_{c-1,2} & \bullet & \bullet & v_{c-1, m} \\
v_{c, 1} & v_{c, 2} & \bullet & \bullet & v_{c, m} \\
\bullet & v_{c+1,2} & \bullet & \bullet & v_{c+1, m} \\
v_{c+r_{1}, 1} & \bullet & & \bullet \\
& v_{c+r_{2}, 2} & & \bullet \\
& & & \bullet & \bullet \\
& & & v_{c+r_{m}-1, m} \\
& & & v_{c+r_{m}, m}
\end{array}\right],
$$

where $l=2 r_{j}+1=2 h_{\mathrm{M} j} / \Delta h_{\mathrm{A}}+1$ is the number of selected field values (number of rows) at the amplitude $h_{\mathrm{M} j}$ for the selected field step $\Delta h_{\mathrm{A}}, c=r_{m}+1$ and $m$ denotes the total number of field amplitudes $h_{\mathrm{M} j}$ (number of columns). Filling the $v_{i j}$-elements of the $V$-matrix from the recorded envelopes of the $\mathrm{BN}$ is performed by an interpolation function for the specified field step $\Delta h_{\mathrm{A}}$. All $V$-matrices belonging to $n$ different values of a structural parameter $z$ are then collected and the arrays $A_{i j}(k=1,2, \ldots n)=$ $\left[v_{i j}(1) / v_{i j}(1), v_{i j}(2) / v_{i j}(1), \ldots, v_{i j}(n) / v_{i j}(1)\right]$ are arranged for all $v_{i j}$-elements. If the $v_{i j}$-elements are inversely proportional to the structural parameter, then reciprocal quantity $1 / v$ is chosen to arrange the $A_{i j}$-arrays. The relative sensitivity of each $v_{i j}$-element to the structural parameter is then determined using a polynomial curve fitting function $p=$ polyfit from a set of the Octave functions [12]. Using this function we find, for every $A_{i j}$-array, a coefficient at linear term of a first degree polynomial that fits the data, $p(z(k))$ to $A_{i j}(k)$, in a least squares sense. The coefficients of all $A_{i j}$-arrays are then written in a sensitivity matrix $S$ of the form of Eq. (2) with $s_{i j}$-elements and the largest element $\max (S)$ is searched for. The indices of this matrix maximum are then the indices of the optimum $\left(h_{\mathrm{A} i}, h_{\mathrm{M} j}\right)$-field pair, at which it is advisable to test the material for particular industrial or other application.

\section{Measurement system}

The scheme of a system designed for measurement of sets of BN signals is shown in Fig. 2. The system operates under control of a personal computer (PC) and its frequency range is $0.1-10 \mathrm{~Hz}$. The power amplifier can provide a maximum output voltage of $30 \mathrm{~V}$ and a maximum current of $2 \mathrm{~A}$. The $\mathrm{BN}$ is measured by a sensor coil wound directly around the sample. The samples are magnetized by a coil wound on the sensing coil. The field value $h_{\mathrm{A}}$ is calculated from the measured magnetizing current. By applying a triangular current to the magnetizing coil we obtain a triangular waveform field with stepwise increasing amplitudes. The field sweep rate is fixed in all triangles and its value was $\pm 4 \mathrm{kA} / \mathrm{m} / \mathrm{s}$ in our experiments. The BN is amplified and filtered from the signal of the sensing coil using an analogue band-pass filter with the cut-off frequencies $1 \mathrm{kHz}$ and $100 \mathrm{kHz}$ and then fed to a data acquisition card.

The PC controls the function generator and collects the data measured by the data acquisition card. The 


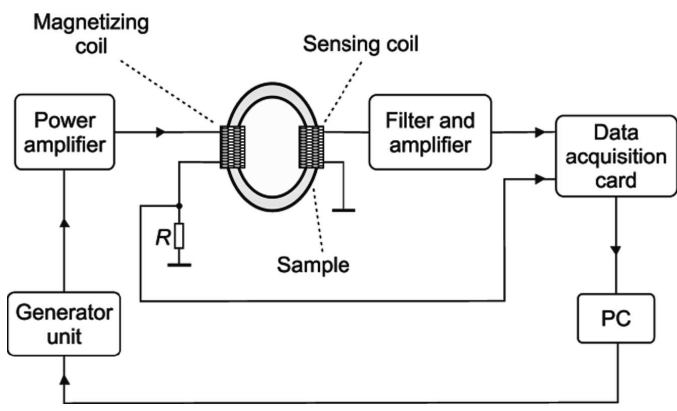

Fig. 2. The principal scheme of the measuring setup.

computer program that acquires and processes the measured signals was developed using the visual engineering environment (VEE) programming tool. Different parameters such as the type of the excitation waveform, initial and final amplitudes of the field, step of the amplitudes and the field rate can be configured by the user interface of the program. The configured excitation waveform is downloaded into the arbitrary waveform generator.

Before each measurement of a set of BN signals, the sample is demagnetized with a sinusoidal signal whose amplitude exponentially decreases to a value close to zero. The initial amplitude and frequency of the demagnetizing signal can be set by the user interface of the program. In each measurement, two signals are acquired by the inputs of the data acquisition card: the voltage induced in the sensing coil and the voltage on the resistor connected in series with the magnetizing coil, proportional to the current flowing through the magnetizing coil. The voltage induced in the sensing coil is used to obtain the $\mathrm{BN}$, whereas the field is calculated from the voltage drop on the resistor.

The low frequency component of the sensing coil voltage is additionally eliminated using the digital sixth-order Butterworth high-pass filter. It is necessary to choose a correct cut-off frequency of the digital filter to sufficiently suppress the low frequency signal [13]. The choice of the cut-off frequency depends on the magnetizing frequency, which was in our measurements in a range of $1-10 \mathrm{~Hz}$. The cut-off frequency of the filter chosen in this work was $1 \mathrm{kHz}$. From the filtered BN, the envelope is calculated using Eq. (1).

The background noise of the BN measurement system was determined as the maximum value of the digitally filtered noise without applying any magnetizing current. Its value is $5 \mu \mathrm{V}$ and the amplitude of the field, which produced the $\mathrm{BN}$ with more than 10 times higher amplitude than this background noise, was used as the initial amplitude of the field in the present measurements.

Finally, the program displays the $\mathrm{BN}$ and stores it in one data file for each measured set of the BN. Each file is processed by a data-evaluation program into one $V$-matrix corresponding to one structural stage of the sample. The sensitivity of the mutually corresponding matrix elements $v_{i j}$ with respect to the investigated ma- terial property is then calculated and the most sensitive elements are searched for. If another type of the $\mathrm{BN}$ parameter is required to evaluate the sensitivity, the columns with the $\mathrm{BN} v_{\mathrm{B}}$ from the files can be processed to obtain it.

\section{Testing the method}

An example of application of the method is shown in this section. The investigated material was commercial low-carbon steel with composition $(\mathrm{C}=0.03, \mathrm{Mn}=0.19$, $\mathrm{Si}=0.13, \mathrm{P}=0.027, \mathrm{~S}=0.027, \mathrm{~N}=0.007 \mathrm{wt} \%)$, grain size of about $50 \mu \mathrm{m}$, and very small dislocation density in undeformed state. Window-shaped samples with different plastic deformation state (engineering tensile strain $\varepsilon=3,6,9,12$, and $26 \%$ ) were used for testing [14]. Typical BN envelopes for triangular driving field with a constant amplitude are shown in Fig. 3.

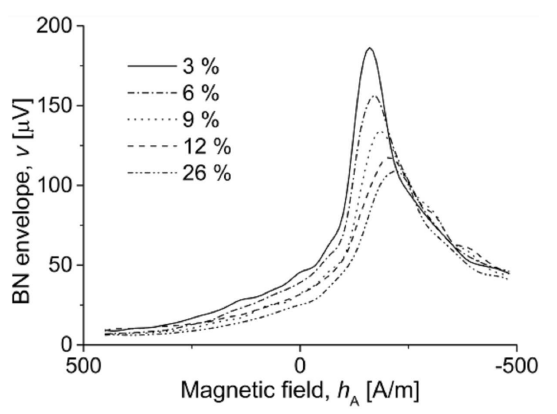

Fig. 3. The BN envelopes for different strains of the low-carbon steel obtained from measurement with pauses between periods of the field at a field amplitude of $500 \mathrm{~A} / \mathrm{m}$.

The initial field amplitude $h_{\mathrm{M} 1}$, maximum value of the amplitude $h_{\mathrm{M} m}$ and step of the amplitude $\Delta h_{\mathrm{M}}$ used in measurements were $100 \mathrm{~A} / \mathrm{m}, 1 \mathrm{kA} / \mathrm{m}$, and $100 \mathrm{~A} / \mathrm{m}$, respectively. The recorded data files obtained from measurements were processed by a data-evaluation program written in the Octave numerical computing environment, which extracted from files the $\mathrm{BN}$ envelope as function of the fields $h_{\mathrm{A}}$ and $h_{\mathrm{M}}$ to evaluate the sensitivity of the $\mathrm{BN}$ to the structural parameter (strain). The program interpolated the $\mathrm{BN}$ envelopes into $V$-matrices with a selected field step $\Delta h_{\mathrm{A}}=50 \mathrm{~A} / \mathrm{m}$. The relative sensitivities $s_{i j}$ of the $v_{i j}(\varepsilon)$-elements for measurement without pauses between periods of the field in $2 \mathrm{D}$ and $3 \mathrm{D}$ plots are presented in Fig. 4a and b, respectively.

We also made comparative measurements of the $\mathrm{BN}$ with pauses between periods of the field. In this case, the $\mathrm{BN}$ was not measured continuously for all amplitudes of the field, but after each period of the magnetizing signal the measurement of the BN was stopped and the sample was demagnetized. The relative sensitivities $s_{i j}$ of the $v_{i j}(\varepsilon)$-elements in $2 \mathrm{D}$ and $3 \mathrm{D}$ plots for this type of magnetizing are displayed in Fig. 5a and b, respectively.

Finally, we compared the optimum $v_{i j}$-elements obtained after adapting the method to the investigated material and property with the traditional RMS value of the 

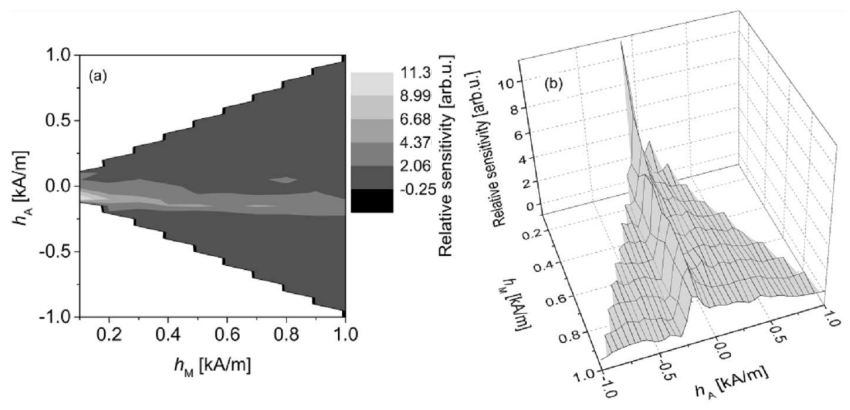

Fig. 4. The 2D (a) and 3D (b) maps of the relative sensitivity $s_{i j}$ of the $v_{i j}(\varepsilon)$-elements obtained from measurement without pauses between periods of the field.

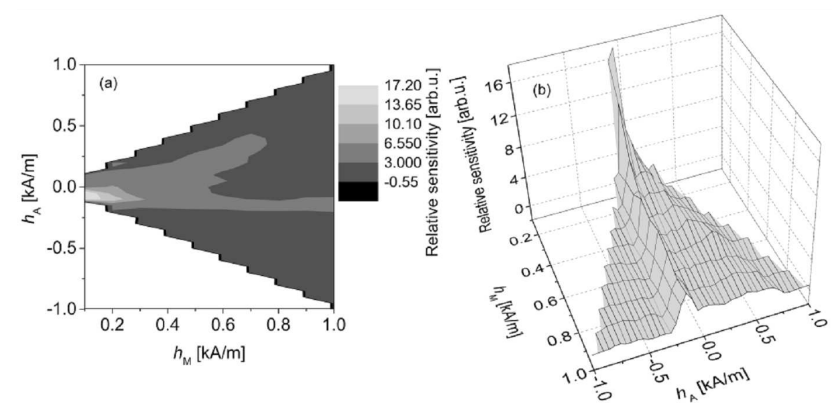

Fig. 5. The 2D (a) and 3D (b) maps of the relative sensitivity $s_{i j}$ of the $v_{i j}(\varepsilon)$-elements obtained from measurement with pauses between periods of the field.

BN measured at a high field. The reciprocal strain dependences of the most sensitive elements $v_{i j}(\varepsilon)$, as well as of the RMS values of the BN measured at a field amplitude of $h_{\mathrm{M}}=1 \mathrm{kA} / \mathrm{m}$ for the both magnetization methods are depicted in Fig. 6.

\section{Discussion}

With rising the strain of the low-carbon steel, the height of the BN envelope decreases (see Fig. 3), which can be attributed to the increase of the dislocation density and therefore consequent drop of the number of irre-

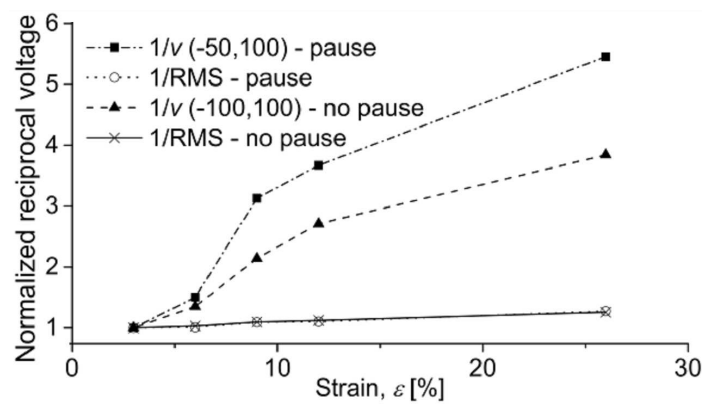

Fig. 6. Normalized reciprocal strain dependences of the most sensitive $v_{i j}(\varepsilon)$-elements $\left(h_{\mathrm{M}}=100 \mathrm{~A} / \mathrm{m}\right)$, and of the RMS values of the $\mathrm{BN}$ at $h_{\mathrm{M}}=1000 \mathrm{~A} / \mathrm{m}$, for measurements with and without pauses between periods of the field. versible magnetization processes [14]. To make the interpretation of the results more convenient, the normalized reciprocal quantities $1 / v$ and $1 / \mathrm{RMS}$ were chosen to compare different methods of the BN processing in Fig. 6 . Since the dependence on the strain $\varepsilon$ is approximately linear in the sensitive part of the envelope, the sensitivity of the $V$-matrix elements to the strain was simply calculated from the slopes of the linear regression lines of the $1 / v(\varepsilon)$ functions. In more complicated situations, when these functions differ from straight lines too much, it is necessary to determine the sensitivity using a more complex method.

From the maps of the relative sensitivity of the $V$-matrix elements to the strain shown in Figs. 4 and 5 it is clear that the most sensitive region is at smallest negative fields. This peak of the sensitivity is consistent with the increase of the differential permeability at small fields [14]. Especially two points $\left(h_{\mathrm{A}}=-50 \mathrm{~A} / \mathrm{m}\right.$, $h_{\mathrm{M}}=100 \mathrm{~A} / \mathrm{m}$ for measurement with pauses between periods of the field) and $\left(h_{\mathrm{A}}=-100 \mathrm{~A} / \mathrm{m}, h_{\mathrm{M}}=100 \mathrm{~A} / \mathrm{m}\right.$ for measurement without pauses between periods of the field) stick out on the graphs. The most sensitive elements of the plots are much more sensitive than the traditional RMS value of the BN (see Fig. 6). This means that adapting the method of the $\mathrm{BN}$ measurement to the investigated low-carbon steel and to the investigated property (strain), we were able to significantly increase the sensitivity of measurement in comparison to traditional measurements. The optimum field amplitudes are much smaller than the maximum value of the amplitude $h_{\mathrm{Mm}}$, which is advantageous with respect to demands on the excitation part of the system. These results are similar as to the MAT utilizing the measurement of differential permeability.

By comparing both magnetization methods in Figs. $4-6$ we can see that they yield somewhat different sensitivity to the strain caused by different initial magnetic state of the materials. Further, we proved that the usage of continuous measurement of the BN without pauses between individual periods of the field takes advantage of the shorter and simpler measurement, but it allows to measure only a limited number of field amplitudes due to the limited memory of data acquisition system.

The field amplitude step used in the presented examples was $\Delta h_{\mathrm{M}}=100 \mathrm{~A} / \mathrm{m}$. It is always a compromise between the technical demands and sensitivity of the BN to structural changes. Smaller step leads to increase of the noise of the results and the demands on the measuring time, size of the storage for the data as well as the data processing time, but it could enhance the sensitivity of the $V$-matrix elements to structural changes. In our examples it was experimentally proven that field amplitude step smaller than $100 \mathrm{~A} / \mathrm{m}$ brings only a slight increase of the sensitivity. The similar rise of the sensitivity can be achieved by decreasing the field step $\Delta h_{\mathrm{A}}$.

To be the adaptive method using the BN successful, several conditions should be met. One important con- 
dition is identical starting magnetic state of the material for all the measurements, which are to be compared with each other. In our method it was achieved by demagnetizing the samples before measuring each set of the BN. Another important condition is that the sample field evaluated from the measurement should not differ in significant way from the real sample field. In the illustrative example of this paper, the magnetically closed samples were used. Closed samples are preferable with respect to ease of assessing the field in the sample. However, magnetically open objects predominate in practical situations of BN measurements, which brings problems of assessing the sample field due to the issues connected with the shape and quality of the magnetic circuit. The sample field in this case should be determined with special care [15]. Further, the parameters of BN depend on field sweep speed, as was shown for example in [16]. This should be kept in mind when measuring the $\mathrm{BN}$, since the magnetic viscosity can seriously distort the results.

The method described in this paper was primarily developed to systematically collect the $\mathrm{BN}$ measurements at different amplitudes of the field. As mentioned above, arranging the data into matrices and their processing described in this paper are close to that used in the MAT. In the proposed method, after collecting the data into $V$-matrices, we are also searching for the most sensitive element of the matrices. When we find it, we can say that the method has been adapted to the investigated material and to the investigated structural property. As a results, the method yields $\left(h_{\mathrm{A} i}, h_{\mathrm{M} j}\right)$-field pair of the most sensitive $V$-matrix element. In general, the method is able to respond to different types of structural changes by adapting to them.

The BN columns of the recorded files contain more information than the BN envelope columns. From the BN columns all possible variables can be computed at any time after the measurement, not only the envelope, which lacks mainly the frequency description of the BN. However, the envelope derived from the BN during the measurement can be used immediately to evaluate structural changes of materials. The actual choice of the variable to be used in a particular case then depends mainly on the practical demands of the measurement, as well as on the compromise between the sensitivity and reliability of results.

\section{Conclusions}

A systematic measurement of the BN at different field amplitudes is proposed in this paper. Magnetization processes are influenced by structural properties of the material. This influence can be higher in some parts of the magnetization processes than in others, which leads to the idea of measuring the $\mathrm{BN}$ at different field amplitudes and collecting these measurements into the data files and matrices of elements, similarly as in the MAT, where the measured quantity is the differential permeability. The recorded data files can be later used for instance for theoretical analysis of the material properties at all meaningful conditions of measurement, even in cases when the samples are no longer available. One part of the recorded data is the $\mathrm{BN}$ envelope, which can be used in practical applications for immediate evaluation of structural changes, when the high sensitivity is needed. The matrices of the BN envelopes and the new magnetization approach were proposed for this purpose. The most sensitive matrix elements can be searched for and those found can be used as indicators of the material structure changes. So the BN envelope column of the recorded files can be used for adapting the measurement to the investigated material and its properties. In the sample measurement of the strain changes of the low-carbon steel, it was confirmed that sensitivity of the $\mathrm{BN}$ to structural changes can be increased in comparison to RMS measurements by choosing the optimum applied field $h_{\mathrm{A}}$ and field amplitude $h_{\mathrm{M}}$. By theoretical analysis of the results, we ascribed the decrease of the BN with increasing strain of the low-carbon steel to the dislocation density increase.

\section{Acknowledgments}

This work was supported in part by the Slovak Research and Development Agency under the contract No. APVV-0062-11, and by VEGA grant VG-1/1325/12.

\section{References}

[1] T. Liu, H. Kikuchi, Y. Kamada, K. Ara, S. Kobayashi, S. Takahashi, J. Magn. Magn. Mater. 310, e989 (2007).

[2] J. Anglada-Rivera, L.R. Padovese, J. Capó-Sánchez, J. Magn. Magn. Mater. 231, 299 (2001).

[3] K. Gurruchaga, A. Martinez-de-Guerenu, M. Soto, F. Arizti, IEEE Trans. Magn. 46, 513 (2010).

[4] J. Blachnio, J. Dutkiewicz, A. Salamon, Mater. Sci. Eng. A 323, 83 (2002).

[5] C. Mandache, T.W. Krause, L. Clapham, IEEE Trans. Magn. 43, 3976 (2007).

[6] I. Tomáš, J. Magn. Magn. Mater. 268, 178 (2004).

[7] I. Tomaš, G. Vértesy, in: Nondestructive Testing Methods and New Applications Ed. M. Omar, InTech, Rijeka, Croatia 2012, p. 145.

[8] G. Vértesy, I. Tomáš, I. Mészáros, J. Magn. Magn. Mater. 310, 76 (2007).

[9] G. Vértesy, I. Tomáš, S. Takahashi, S. Kobayashi, Y. Kamada, H. Kikuchi, NDT E Int. 41, 252 (2008).

[10] B. Skrbek, I. Tomáš, J. Kadlecová, N. Ganev, Kovove Materialy - Met. Mater. 49, 401 (2011).

[11] G. Vértesy, I. Tomáš, T. Uchimoto, T. Takagi, NDT E Int. 47, 51 (2012).

[12] J. Schmidt Hansen, GNU Octave Beginner's Guide, Packt Publishing, Birmingham 2011.

[13] J. Pal'a, J. Bydžovský, J. Electr. Eng. 63, 106 (2012).

[14] J. Pal'a, O. Stupakov, J. Bydžovský, I. Tomáš, V. Novák, J. Magn. Magn. Mater. 310, 57 (2007).

[15] O. Stupakov, J. Magn. Magn. Mater. 307, 279 (2006).

[16] L. Batista, U. Rabe, I. Altpeter, S. Hirsekorn, G. Dobmann, J. Magn. Magn. Mater. 354, 248 (2014). 